\title{
Assessment of aerodynamic noise in an industrial ventilation system
}

\author{
A. M. Martins \& A. C. Mendes \\ Universidade da Beira Interior, \\ Laboratory of Fluid Mechanics and Turbomachinery, Covilhã, Portugal
}

\begin{abstract}
This paper deals with the assessment of aerodynamic noise level generated by an industrial ventilation system. The system incorporates an axial flow fan operating with airfoil rotor blades. Measurements were performed using a modular digital sonometer of high precision, equipped with a microphone and a frequency analyser. The procedure that was followed enabled us to account for both the global level and the equivalent frequency level of noise, in the intermediate range of audible frequencies, using a filter of $1 / 3$ of the octave.

Keywords: aerodynamic noise, axial flow fans, industrial ventilation systems.
\end{abstract}

\section{Introduction}

Sound is a form of energy associated with the vibration of material particles in a medium. The displacements of the oscillating particles are transferred throughout the matter as acoustic energy, which travels under the form of a sound wave. Acoustics is the science that studies the generation and transmission of such sound waves. It concerns not only the phenomenon that occurs in air, which is audible to humans, but also other phenomena governed by the same basic laws. Sound may as well be transmitted through solids, liquids and gases. In all cases we are mainly concerned with the study of a wave motion which is, however, very distinct from the motion of the individual particles in the medium. Sound is effectively a mechanical wave motion whose propagation depends on the physical properties of matter. To be transmitted, sound relies on the elasticity and inertia of the material in question [1].

Noise, on the other hand, is a sonorous stimulus that is unpleasant to our hearing and without significant information to its receptor. It can become nevertheless an issue of primary importance in what refers to human health and 
environmental quality. Noise will be caused by a number of factors, for instance by friction between mechanical components, like in bearings and gears, by the vibration induced by floating masses in internal combustion engines, or even by cavitation induced vibrations in hydraulic pumps and turbines. Eventually, the noise arising in industrial equipment will be caused by the same factors that contribute to its loss of efficiency. Therefore, it can be expected that a gain in the performance of any machine will result in a lower level of noise in the installation [2].

In the specific case of fans and compressors, aerodynamic instabilities play a decisive role in the global noise level of the system. Aerodynamic noise, as is usually called, is mainly caused by separation of boundary layer flow at the upper surface of the rotor blades [3]. The noise level can be significant, especially in axial-flow compressors operating close to stall conditions. Another important cause of aerodynamic noise in axial machines is vortex shedding from the blades trailing edge. The noise frequency depends, in this case, essentially upon the blade profile and velocity. As this velocity varies along the blade, the noise generated by the interaction between the rotor and working fluid covers a wide range of frequencies. In the case of rotors presenting a great number of blades, another relevant source of noise appears to be the interaction between the wake of one blade with the adjacent one. These interactions become more important as the rotational speed of the machine increases.

An inadequate tip clearance between the rotor of an axial-flow machine and its casing may also be at the origin of noise production and loss of efficiency. This is essentially associated with undesired secondary flow effects [4]. Moreover, turbulent flow is also an important cause of noise, in particular for airflow velocities higher than $5 \mathrm{~m} / \mathrm{s}$.

Finally, the stability of the entire ventilation system is crucial in what concerns noise level. Vibrations induced by the rotor upon its casing and adjacent ducts may become intense, especially near structural resonance.

In order to measure the noise that is generated by ventilation systems it is often used a sonometer of high precision. In a first step the equivalent continuous sound level of the installation is usually assessed. However, this parameter in itself is not a sufficient indication of what is felt by the human hearing. In reality we are more sensitive to sound frequencies between $500 \mathrm{~Hz}$ and $4 \mathrm{KHz}$. On the other hand, sound frequencies lower than $20 \mathrm{~Hz}$ or higher than $20 \mathrm{KHz}$ become unperceptive. Consequently, it is also necessary to perform measurements of the equivalent sound level in frequency. For that purpose sound meters usually include a frequency analysis module, with a filter of an octave or $1 / 3$ of the octave band, for the frequencies belonging to the audible frequency bandwidth. Measurements requiring a good precision must inclusively be conducted in an anechoic test facility [5].

The present work deals with the assessment of aerodynamic noise level generated by an industrial fan equipped with airfoil rotor blades. Measurements were performed using a modular digital sonometer of high precision. The procedure followed enables us to present herein the equivalent continuous sound 
level and the equivalent frequency level of noise generated by the fan, in the intermediate range of audible frequencies, using a filter of $1 / 3$ of the octave.

\section{Experimental apparatus}

An industrial fan currently under investigation at the Turbomachinery Laboratory of UBI [6] has been tested in the axial-flow test bench (Fig. 1). The experimental apparatus consists of a tubular duct that is fully instrumented in view of making the performance analysis of axial-flow fans. Below we describe this experimental facility.

\subsection{Test bench}

The main body of the test facility is a four-element steel duct having a circular cross-section. These four elements are assembled together with the casing of the fan to be tested. The location of the machine is directly related to obtaining uniform flow conditions at the inlet of the rotor. A valve placed at the outlet section of the duct, downstream of the fan, controls the flow rate. The velocity of the flow is measured by a telescopic Prandtl tube, positioned upstream of the rotor. The data acquired by the probe covers the width of the bench crosssection. The axial mean velocity of the flow is assessed by another Prandtl probe, which in turn is placed downstream of the machine. The head rise of the working fluid across the rotor is measured at eight static pressure inlets, four of these before and four after the fan rotor. Static and total pressure heads are red in $\mathrm{mm}$ of alcohol, by means of a U-tube panel having a variable inclination. The rotational speed of the rotor is measured with the help of a tachometer. A digital thermometer and a barometer monitor the atmospheric temperature and pressure.

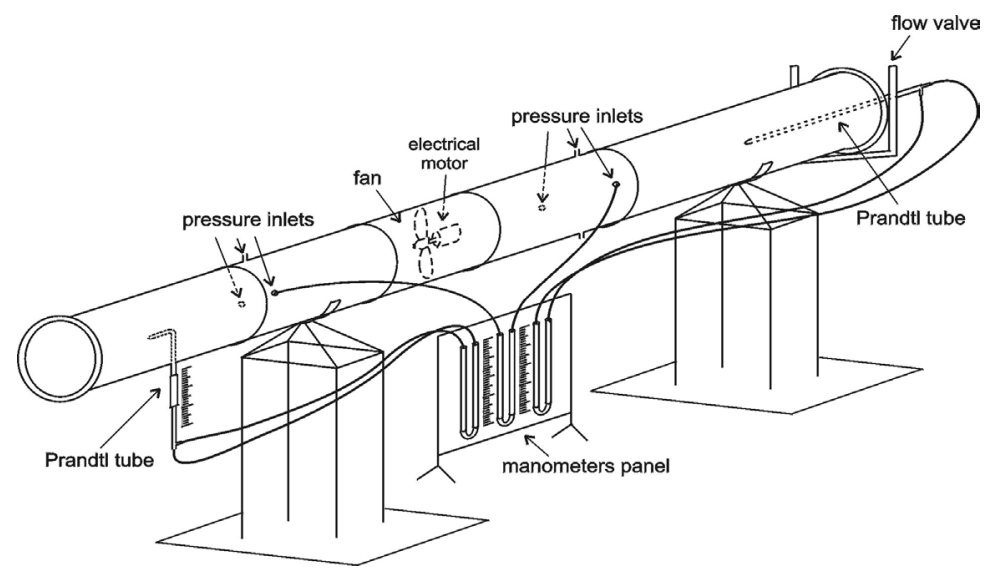

Figure 1: Axial-flow fans test bench. 


\subsection{Axial-flow fan}

The machine to be tested is an axial-flow fan whose rotor is driven by an electrical motor. The machine is mounted in its circular casing and later on assembled to the test bench (see Fig. 2).

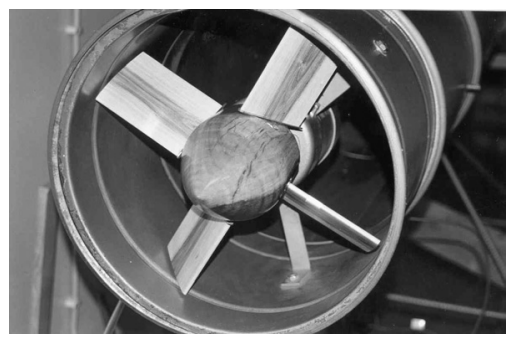

Figure 2: Axial-flow fan under investigation.

In order to obtain a flexible installation, the hub is able to accommodate different rotor blades with a pre-defined geometry. Two sets of blades have been produced, all manufactured in wood. The axial-flow fan that makes the object of the present study was equipped with four blades especially adapted to working at low Reynolds Number. In nominal conditions for this machine $\mathrm{Re}$ is of the order of $5 \times 10^{4}$. Tab. 1 summarises the characteristics of the fan rotor. The angle of attack of the blade wing sections may be conveniently controlled, in order to get the best flow entry at the leading edge. On the other hand, the hub was designed in such a way that it offsets the electrical motor and, hence, reduces the overall resistance associated with the central core of the machine.

Table 1: Geometrical characteristics of the fan rotor.

\begin{tabular}{|lr|}
\hline Rotor diameter $(D)$ & $295[\mathrm{~mm}]$ \\
\hline Hub diameter $\left(d_{c}\right)$ & $101,6[\mathrm{~mm}]$ \\
\hline Number of blades $(n)$ & 4 \\
\hline Blade length $(l)$ & $98[\mathrm{~mm}]$ \\
\hline Blade chord $(c)$ & $70[\mathrm{~mm}]$ \\
\hline Pitch at mean radius $(t)$ & $193[\mathrm{~mm}]$ \\
\hline Pitch-chord ratio at mean radius $(t / c)$ & 2,76 \\
\hline Maximal blade thickness & $9,35 \%$ of the chord \\
\hline X-coordinate of maximal thickness point & $26,3 \%$ of the chord \\
\hline Maximal y-coordinate of the camber line r & $3 \%$ of the chord \\
\hline $\begin{array}{l}\text { X-coordinate of inflexion point at the upper surface } 39,8 \% \text { of } \\
\text { the chord }\end{array}$ & $13\left[^{\circ}\right]$ \\
\hline Camber angle at leading edge $\left(\theta_{1}\right)$ & $8\left[^{\circ}\right]$ \\
\hline Camber angle at the trailing edge $\left(\theta_{2}\right)$ & $21\left[^{\circ}\right]$ \\
\hline Camber line angle $(\theta)$ & \\
\hline
\end{tabular}


In order to conduct the performance analysis of the fan, the machine was firstly inserted in the test bench and driven at a rotor speed of $N=1466 \mathrm{rpm}$. The stagger angle of the blades was selected at $\lambda=48^{\circ}$. Total head across the rotor was then measured for different working conditions, as the flow rate is varied by means of the valve at the end of the experimental facility. Fig. 3 shows the evolution of the internal efficiency $\eta$ of the fan, i.e. the ratio between the hydraulic power and the power available at the shaft of the electric motor. The fan efficiency is here represented as a function of flow rate coefficient $\phi=Q / N D^{3}$.

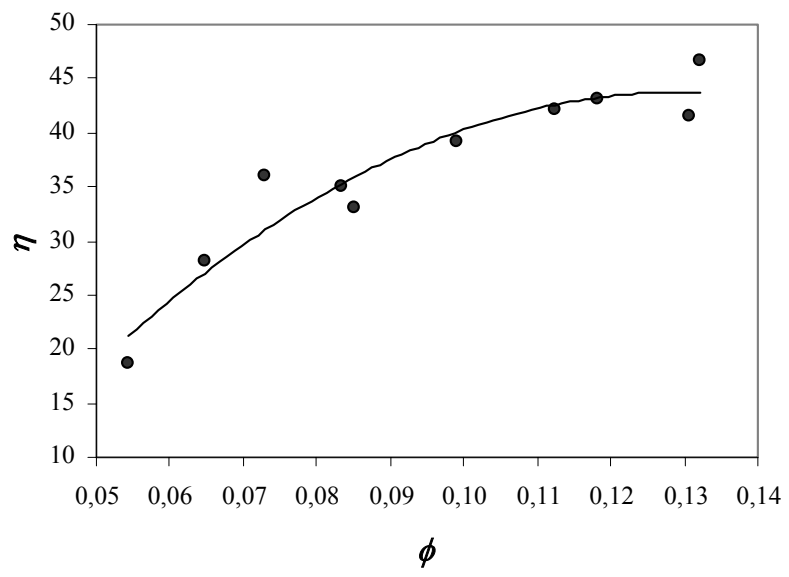

Figure 3: Internal efficiency versus flow-rate coefficient.

The analysis of the curve shows that nominal conditions correspond to the highest values of flow rate, of the order of $Q=510 \mathrm{l} / \mathrm{s}$, for which the fan efficiency is about $\eta=42 \%$. It is expected that noise level will be less significant under these working conditions.

\section{Noise assessment}

In this section we present and discuss the noise measurements that were carried out while the fan was being tested in the ventilation bench. The noise in the ventilation system was measured by means of a modular digital sonometer of high precision, model 2231, produced by Brüel and Kjær [7]. This device is equipped with a microphone (model 4155) and a module for frequency analysis (model BZ 7103). We may print the results of the measurements directly on paper by using the printer of the equipment (model 2318/ZI 0054).

The sonometer was placed at the rear end of the test facility, facing the back of the rotor, as is shown in Fig. 4. Two series of measurements were conducted. Firstly the global level of noise was assessed, while running the fan for different 
flow-rate conditions. After this the level of noise in frequency was assessed at maximal flow-rate conditions, i.e. by keeping the valve at the end of the bench $100 \%$ open. All the measurements were recorded for a rotating speed corresponding to nominal working conditions $(N=1466 \mathrm{rpm})$ and blades stagger angle $\lambda=48^{\circ}$.

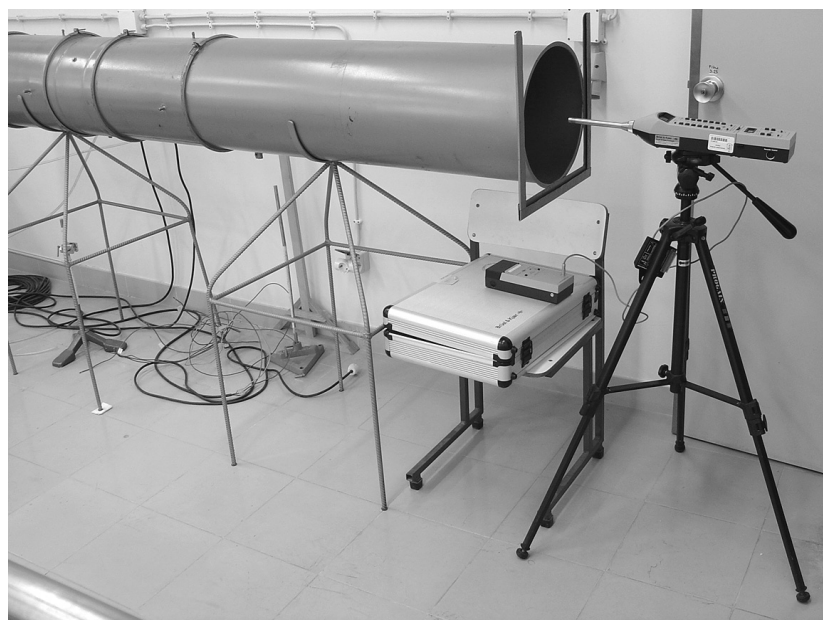

Figure 4: Equipment for noise assessment.

Table 2: $\quad$ Sonometer set-up parameters.

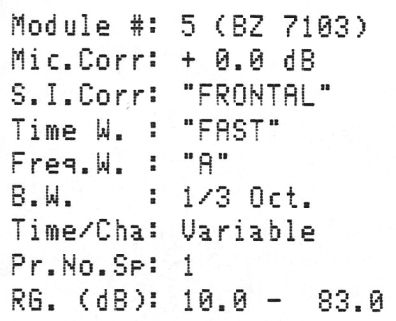

\subsection{Sonometer set-up}

The Table (Tab. 2) shows the set-up parameters that were selected for the sonometer before starting the measurements. S.I.Corr. is associated with the choice of the microphone and its orientation, with respect to the source of noise. This parameter was selected as frontal in accordance with CEI Standards, as the microphone is directed towards the fan rotor. Parameter Time $W$ corresponds to time weighting; it was prescribed as fast, because we are confronted with oscillating noise, which is typical for industrial equipment. Freq $W$ is the frequency weighting parameter, here defined as $A$, according to CEI 651 
Standard. B.W. parameter determines the frequency step at which noise levels are recorded; in the present case we have assumed $1 / 3$ of the octave, for the central frequencies of the audible range ( $20 \mathrm{~Hz}$ to $20 \mathrm{kHz})$. Time/Cha parameter, defined internally by the sonometer as variable, is the time interval for data acquisition in each of the considered frequencies. Finally $R G$ (in $\mathrm{dB}$ ) defines the range of values for $L_{e q, f}$, i.e. the minimal and maximal values of the sound power level.

\subsection{Equivalent continuous sound level}

The equivalent continuous sound level $\left(L_{e q}\right)$ is the temporal mean of sound pressure level $(S P L)$, taken during the time interval $T$ :

$$
L_{e q, T}=10 \log _{10} \frac{1}{T} \int_{0}^{T}\left(\frac{p(t)}{p_{r}}\right)^{2} d t
$$

Here $p_{r}$ is the sound pressure of reference $(20 \mu \mathrm{Pa}), p(t)$ is the sound pressure measured as a function of time, and $T$ is the period of measurement. Fig. 5 presents the values of $L_{e q}$ that have been recorded placing the microphone at the end of the ventilation duct. They are represented as a function of $\phi$. These values were obtained for the fan nominal working speed, by varying the flowrate valve between $50 \%$ and $100 \%$ of the duct opening, and taking $T=30 \mathrm{~s}$.

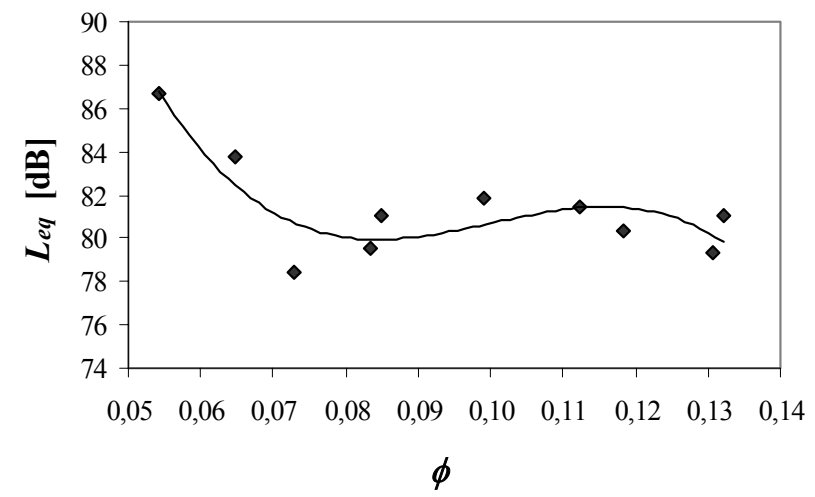

Figure 5: Equivalent continuous sound level as a function of flow-rate coefficient.

As we can observe, the range of the fan nominal working conditions coincides with the minimum values of $L_{e q}$. On the other hand, for reduced values of flow-rate the noise level increases exponentially. This corresponds in fact to a recognizable zone of instability for this type of machine. 


\subsection{Equivalent sound level at frequency $f$}

Another important parameter in this context is the equivalent sound level at frequency $f\left(L_{e q, f}\right)$. This parameter represents the mean value of the sound pressure level at each of the sampling rate frequencies, which are the central frequencies of the standard $1 / 3$ octave band. Such a parameter is important for the identification of frequency bands for which the sound pressure level is higher than the equivalent continuous sound level.

In this series of tests the sonometer was placed once again at the outlet of the test bench, facing the rear of the fan. $L_{e q, f}$ was then recorded for the central frequencies of $1 / 3$-octave of the audible spectrum, between $20 \mathrm{~Hz}$ and $20 \mathrm{kHz}$. Tab. 3 shows the values that were printed by the frequency analyser of the sonometer.

Table 3: Equivalent sound level at frequency $f$, for $\lambda=48^{\circ}$ and $N=1466 \mathrm{rpm}$.

\begin{tabular}{|c|c|c|c|}
\hline $\begin{array}{c}\text { Frequency } \\
{[\mathbf{H z}]}\end{array}$ & $\boldsymbol{L}_{\boldsymbol{e q}, f}[\mathbf{d B}]$ & $\begin{array}{c}\text { Frequêncy } \\
{[\mathbf{H z}]}\end{array}$ & $\boldsymbol{L}_{\boldsymbol{e q}, \boldsymbol{f}}[\mathbf{d B}]$ \\
\hline 20 & $50,0 \pm 0,1$ & 800 & $75,8 \pm 0,1$ \\
\hline 25 & $51,8 \pm 0,1$ & 1000 & $75,3 \pm 0,1$ \\
\hline 31,5 & $54,4 \pm 0,1$ & 1250 & $74,8 \pm 0,1$ \\
\hline 40 & $56,3 \pm 0,1$ & 1600 & $74,1 \pm 0,1$ \\
\hline 50 & $58,7 \pm 0,1$ & 2000 & $72,4 \pm 0,1$ \\
\hline 63 & $60,2 \pm 0,1$ & 2500 & $70,7 \pm 0,1$ \\
\hline 80 & $63,7 \pm 0,1$ & 3150 & $69,3 \pm 0,1$ \\
\hline 100 & $66,9 \pm 0,1$ & 4000 & $67,2 \pm 0,1$ \\
\hline 125 & $69,3 \pm 0,1$ & 5000 & $64,4 \pm 0,1$ \\
\hline 160 & $71,6 \pm 0,1$ & 6300 & $62,0 \pm 0,1$ \\
\hline 200 & $72,7 \pm 0,1$ & 8000 & $58,2 \pm 0,1$ \\
\hline 250 & $74,8 \pm 0,1$ & 10000 & $55,2 \pm 0,1$ \\
\hline 315 & $74,9 \pm 0,1$ & 12500 & $51,7 \pm 0,1$ \\
\hline 400 & $75,4 \pm 0,1$ & 16000 & $47,8 \pm 0,1$ \\
\hline 500 & $75,0 \pm 0,1$ & 20000 & $45,6 \pm 0,1$ \\
\hline 630 & $75,8 \pm 0,1$ & & \\
\hline
\end{tabular}

As it can be observed for this range of frequencies the level of noise in the installation varies between $45,6 d B$ and $75,8 d B$. Moreover, the maximal values appear to occur in the frequency range $f=200 \mathrm{~Hz}-2 \mathrm{kHz}$. We may notice that the sound level presents a smooth evolution in the entire range of the audible frequencies. 


\section{Conclusions}

Most of the aerodynamic noise that is generated by industrial axial-flow fans is propagated to the environment via the inlet and outlet ends of the ventilation system. In reality, less than $5 \%$ of this noise is propagated directly through the walls of the ventilation duct. On the other hand, noise transmission along the ventilation ducts is quite efficient, unless appropriate noise dissipaters are used. The design of such devices, however, must take into consideration that no significant head losses are to be introduced in the system.

One way to meet this purpose is to use noise absorbent materials to isolate the duct and overall system housing. It is an expensive technique that gains importance nowadays, especially in large factories, where the level of noise is sometimes so high that any small reduction is important, in terms of human comfort. This solution is equally applicable in auditoriums and public theatres, where noise should be unperceptive and a constant ventilation of the space is nevertheless indispensable. In the case of axial machines this technique is particularly useful to mitigate noise at frequencies above $500 \mathrm{~Hz}$ [8].

By comparing our results with other results published for industrial axial-flow fans [9], we may conclude that they are of the same order of magnitude of those obtained for fans with similar characteristics, at least in what concerns the equivalent continuous sound level. The frequency analysis of sound is, however, of fundamental importance when we wish to reduce noise levels that were not detected in measurements of equivalent continuous sound level. For the central frequencies of $1 / 3$-octave of the audible spectrum, the level of noise in our installation varied between $45,6 \mathrm{~dB}$ and $75,8 \mathrm{~dB}$, with its maximal values in the frequency range $f=200 \mathrm{~Hz}-2 \mathrm{kHz}$. We have also noticed that the sound level presents a smooth evolution in the entire range of the audible frequencies.

Another important source of noise could be the structural vibration that is induced by the fan on the ventilation system, particularly if near resonance. The ducts should be well fixed and the use of appropriate supporting elements, capable of damping these induced vibrations, should be considered.

\section{Acknowledgements}

The present work was carried out at the Laboratory of Fluid Mechanics and Turbomachinery of Universidade da Beira Interior, in Portugal. The authors are indebted to the Aerospace Sciences Department of UBI, for the use of their equipment of noise measurement.

\section{References}

[1] Pierce, A.D., Acoustics - An Introduction to Its Physical Principles and Applications, McGraw-Hill Book Co., New York, 1981.

[2] Envia, E., Fan Noise Reduction - An Overview, AIAA-2001-0661, Glenn Research Center, Cleveland, 2001. 
[3] Hay, N., Mather, J. \& Metcalfe, R., Fan Blade Selection for Low Noise, Proc. Seminar of Fluid Machinery Committee, pp. 51-57, Beccles, 1987.

[4] Neuhaus, L. \& Neise, W., Active Flow Control to Reduce the Tip Clearence Noise and Improve the Aerodynamic Performance of Axial Turbomachines, Proc. Fan Noise Int. Symposium, 8 pp., Senlis, 2003.

[5] Jansson, D., Mathew, J., Hubner, P., Sheplak, M. \& Cattafesta, L., Design and Validation of an Aeroacoustic Anechoic Test Facility, Proc. $8^{\text {th }}$ AIAA/CEAS Aeroacoustics Conference, pp. 1-10, Breckenridge, 2002.

[6] Mendes, A.C., Martins, A.M., Marques, B.T. \& Pascoa, J.C., Design and Performance Analysis of a Rotor for an Industrial Axial Flow Fan (in Portuguese), Proc. VI Congresso Ibero-Americano de Engenharia Mecânica, Vol. II, pp. 1531-1536, ed. A. M. Dias, Coimbra, 2003.

[7] Brüel \& Kjær, Modular Precision Sound Level Meter plus Integrating SLM Application Module, Instruction Manual (2231 + BZ 71103), Nærum, Denmark, 1987.

[8] Osborne, W., The Selection and Use of Fans, Engineering Design Guides 33, pp. 1-17, Oxford, 1980.

[9] Soler \& Palau, Industrial Catalog, Spain, 2001. 